\section{МОРФОАОГИЧЕСКИЕ ОСОБЕННОСТИ АИСТААЬНОГО ОТАЕАА АЕГОЧНЫХ ВЕН У БОАЬНЫХ С ФИБРИААЯЦИЕЙ ПРЕАСЕРАИЙ ПО ААННЫМ МУАЬТИСПИРААЬНОЙ КОМПЬЮТЕРНОЙ ТОМОГРАФИИ}

\author{
Хамнагалаев И.А. ${ }^{13}$, Тарбаева Н.В.2 , Булавина И.А. ${ }^{3}$, Термосесов С.А. ${ }^{1,3}$, \\ Ильич И.А. ${ }^{3}$, Хамнагадаев И.И. ${ }^{4}$, Кармазановский Г.Г.5, Калашников В.Ю. 2 , \\ Школьникова М.А. ${ }^{1}$, КОков $\wedge . С .6,7$
}

1 - НИКИ педиатрии ГБОУ ВПО РНИМУ им. Н.И. Пирогова Минздрава России. 2 - ФГБУ «Национальный медицинский исследовательский центр атетерная изоляция мегочных вен (КИАВ) является операцией выбора у паци- эндокринологии" ентов с пароксизмальной формой фибримяяции предсердий (ФП). Предопера- Минздрава России. ционная оценка вариантов впадения мегочных вен $(\Lambda \mathrm{B})$ в мевое предсердие 3 - ГБуз «Городская $(\Lambda П)$ является важным этапом планирования вмешательства. Существующие клиническая больница анатомические классификации вариантов впадения $\Lambda$ В в $\Lambda$, как правило, трудно им. В.М. Буянова"дз применимы в клинической практике.

Москвы.

Цель исследования. Классифицировать варианты дистальной анатомии мёгоч- г. Москва, Россия. ных вен у больных с фибримляцией предсердий по данным мультиспиральной компью- 4 - ФГАОУ ВО терной томографии (МСKT).

"Бемгородский

Материалы и методы. В исследование включено 167 пациентов (80 - женского государственный пола) в возрасте 18-79 лет, которым выполнена МСКТ $\Lambda$ и $\Lambda$ В дмя оценки их анатоми- национальньй ческих особенностей перед выполнением КИАВ. Пароксизмальная форма ФП - 107 исследовательский больных (64,1\%), персистирующая фрорма ФП - 39 пациентов (23,3\%). В 21 случае университет». $(12,6 \%)$ форма ФП не уточнена.

г. Бемгород, Россия.

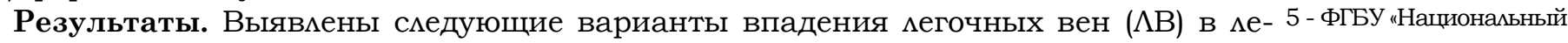
вое предсердие $(\Lambda П)$. Вариант 1 - типичная анатомия: наличие четырех раздельных медицинский устьев $\Lambda \mathrm{B}(\mathrm{n}=88 ; 52,7 \%)$. Вариант 2 - рассыпной тип: наличие трех или более раздемь- исследовательский центр ных ипсилатеральных устьев $\Lambda \mathrm{B}(\mathrm{n}=36 ; 21,5 \%)$. Вариант 3 - комлектор мевых $\Lambda$ В: $е$ евая хирургии им. А.В. верхняя и мевая нижняя $\Lambda \mathrm{B}$ сливаются в один ство до впадения в $\Lambda$ (n=31; 18,6\%). Вишневского" Вариант 4 - смешанный тип: сочетание комлектора $\Lambda$ В с рассыпным типом строения Минздрава России. (n=10; 6\%). Вариант 5 - общий ствол контрлатеральных $\Lambda$ В: слияние контрлатеральных 6 - ГБуз «НИи скорой $\Lambda$ В в один ство до впадения в $\Lambda$ (n=1; 0,6\%). Вариант 6 - комлектор правых $\Lambda$ В (n=1; помощи им. Н.В. $0,6 \%)$.

Заключение. Дистальная анатомия $\Lambda \mathrm{B}$ характеризуется выраженной вариабель- Москвы. ностью. У больных с ФП типичный анатомический паттерн впадения $\Lambda$ В в $\Lambda$ встреча- 7 - ФГАОУ вО «Первый ется в 52,7\% случаев. Реже встречаются рассыпной тип строения $\Lambda$, наличие комлек- МгмУ им. И.М. тора $\Lambda$ В, а также их сочетание. Комлекторы правых $\Lambda$ В и контрлатеральных $\Lambda$ В яв яют- Сеченова" Минздрава ся крайне редкими анатомическими вариантами. Выделение шести вариантов впаде- России (Сеченовский ния $\Lambda$ В в $\Lambda$ (Вариант 1 - типичная анатомия; Вариант 2 - рассыпной тип; Вариант 3 - Университет). комлектор мевых $\Lambda$; Вариант 4 - смешанный тип; Вариант 5 - комлектор контрлате- г. Москва, Россия. ральных $\Lambda$; Вариант 6 - комлектор правых $\Lambda$ В) позволяет описать особенности дистальной анатомии мегочных вен по данным МСКТ. графия.

КАючевые слова: фибримяяция предсердий, мегочные вены, компьютерная томо-

Контактный автор: Хамнагадев И.А., e-mail: i@khamnagadaev.ru

Для иитирования: Хамнагадаев И.А., Тарбаева Н.В., Булавина И.А., Термосесов С.А., Ильич И.А., Хамнагадаев И.И., Кармазановский Г.Г., Калашников В.Ю., Школьникова М.А., Коков А.С. Морфологические особенности дистального отдела легочных вен у больных с фибрилляиией предсердий по данным мультиспиральной компьютерной томографиu. REJR 2019; 9(1):57-66. DOI:10.21569/2222-7415-2019-9-1-57-66.
Статья получена:
13.02 .19
Статья принята:
06.03.19 


\title{
PULMONARY VEIN ANATOMY VARIATIONS IN PATIENTS WITH ATRIAL FIBRILLATION: COMPUTED TOMOGRAPHY STUDY
}

\author{
Khamnagadaev I.A. 1,3, Bulavina I.A.2, Tarbaeva N.V.3, Termosesov S.A. 1,3, llich I.L.3, \\ Khamnagadaev I.I.4, Karmazanovsky G.G.5, Kalashnikov V.Yu.2, \\ Shkolnikova M.A. ${ }^{1}$, Kokov L.S.6,7
}

$\mathrm{P}$

ulmonary vein (PV) isolation is the mainstay of atrial fibrillation (AF) ablation. PV anatomy classifications have been investigated. Despite this they have not been applied to decide the best ablation strategy.

- Research and Clinical

Institute for Pediatrics of

Purpose. To classify the various patterns of the PV anatomy based on multislice computed tomography (CT) evaluation in AF patients.

Methods. CT was performed prior to AF ablation in 167 (80 females) patients aged 18-79 years old, $107(64.1 \%)$ paroxysmal and $39(23.3 \%)$ persistent AF patients, in 21 $(12.6 \%)$ patients type of AF was not identified.

the Pirogov Russian

National Research

Medical University.

2 - National research

Results. Subsequent anatomical patterns have been found. Type I (normal anatomy) - four independent PV with four separate ostia ( $\mathrm{n}=88 ; 52.7 \%)$. Type II (dichotomous pattern) - three or more independent ipsilateral PV ostia ( $n=36 ; 21.5 \%)$. Type III (left common PV ostium) - coalescence of the left superior and left inferior PVs proximal to the left atrium (LA) $(n=31 ; 18.6 \%)$. Type IV (combined pattern) - II and III pattern combination $(n=10 ; 6 \%)$. Type $\mathrm{V}$ (Contralateral PV common ostium) - coalescence of the right and left inferior PVs proximal to the LA ( $\mathrm{n}=1 ; 0.6 \%)$. Type VI (Right common PV ostium) - coalescence of the right PVs proximal to the LA ( $\mathrm{n}=1 ; 0.6 \%)$.

Conclusion. PV anatomy was confirmed to be highly variable. Normal PV anatomical pattern in AF patients has been revealed in 52.7\%. Dichotomous PV pattern, left common ostium presence and these combinations have been found less frequently. Common PV trunk and right common ostium were considered to be extremely rare patterns. These findings are supposed to be useful for appropriate ablation strategy selection.

Keywords: Atrial fibrillation, pulmonary veins, computed tomography.

Corresponding author: Khamnagadaev I.A., e-mail: i@khamnagadaev.ru

For citation: Khamnagadaev I.A., Bulavina I.A., Tarbaeva N.V., Termosesov S.A., Mich I.L., Khamnagadaev I.I., Karmazanovsky G.G., Kalashnikov V.Yu., Shkolnikova M.A., Kokov L.S. Pulmonary vein anatomy variations in patients with atrial fibrillations: computed tomography study. REJR 2019; 9(1):57-66. DOI:10.21569/2222-7415-2019-9-1-57-66.

Received: $\quad 13.02 .19 \quad$ Accepted: $\quad 16.03 .19$

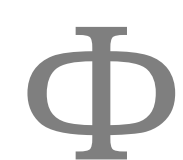

ибримляция предсердий (ФП) в настоящее время явмяется одной из распространенных аритмий, которая диагностируется у $0,4 \%$ насемения земного шара и ассоциирована с высоким риском инвалидизации и смертности вследствие кардиогенных тромбоэмболий, а также развития и декомпенсации хронической сердечной недостаточности [1 - 3]. Одной из ведущих причин возникновения ФП может быть эктопическая активность в мегочных венах $(\Lambda \mathrm{B})$ [2]. Стратегия, направценная на удержание синусового ритма, явцяется более предпочтительной по сравнению с подходом, направленным на контроль частоты сердечных сокращений. В соответствии с рекомендациями Европейского кардиологического общества (2017), катетерная изоляция мегочных вен (КИАВ) может быть ре- комендована в качестве первой минии мечения пациентов с пароксизмальной формой фибрилмяции предсердий [2 - 4].

Эффективность рентгеноэндоваскулярных способов цечения данной патологии остается низкой, приводя к необходимости выполнения повторных вмешательств. По данным Gaita F.

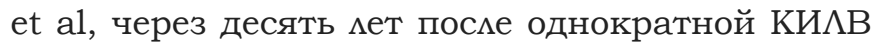
отсутствие рецидивов ФП отмечается только в $39 \%$ случаев при пароксизмальной и в $25 \%$ случаев при персистирующей форме ФП. При выполнении повторных вмешательств десятилетняя эффективность достигает $61 \%$ и 44\% соответственно [5].

Одним из факторов, ассоциированным с результатами катетерной изоляции цегочных вен $(\Lambda \mathrm{B})$, является вариабельность их дистальной анатомии. Выявмено, что у пациентов с не- 
типичной анатомией $\Lambda \mathrm{B}$ частота рецидивирования ФП после КИАВ может быть выше, чем у больных с типичным паттерном впадения $\Lambda \mathrm{B}$ в мевое предсердие $(\Lambda П)$ [6]. Среди нетипичных анатомических вариантов наличие комлектора $\Lambda \mathrm{B}$ ассоциировано с более низкой частотой рецидивирования ФП после КИАВ [7 - 10].

По мнению различных исследователей, предоперационное изучение вариационной анатомии мегочных вен по данным мультиспиральной компьютерной томографии (MCKT) явяяется рациональным подходом [2, 11 - 18]. Несмотря на это, ранее разработанные классификации впадения мегочных вен в мевое предсердие, трудно применимы в клинической практике и не всегда учитывают редкие анатомические варианты.

\section{Цель исследования.}

Классифицировать варианты дистальной анатомии мегочных вен у больных с фибримляцией предсердий по данным мультиспиральной компьютерной томографии.

Материалы и методы.

Характеристика пациентов.

В исследование включено 167 пациентов в возрасте 18-79 мет, которым выполнена МСКТ

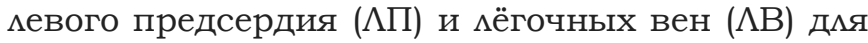
оценки их анатомических особенностей перед выполнением катетерной изоляции мегочных вен (КИ $\Lambda$ В). Медиана $(\mathrm{Me)}$ возраста составица 60 мет, интерквартимьный размах (IQR) - 54-65 мет. Пациентов мужского пома было 87 человек (52,1\%), женского - 80 (47,9\%). Характеристика пациентов приведена в таблице №1.

Критерии вкАючения в исследование: наличие пароксизмальной или персистирующей формы ФП, в случае подготовки пациента к катетерной изоляции $\Lambda \mathrm{B}$.

Критерии исключения из исследования: постоянная форма ФП, фракция выброса мевого желудочка менее 50\%, тяжелая экстракардиальная патология, тромбоз полостей сердца, врожденные и приобретенные пороки сердца, кардиомиопатии, ранее перенесенные операции на открытом сердце, наличие противопоказаний $\mathrm{K}$ введению рентгеноконтрастных средств.

\section{Исследуемые параметры.}

Во всех случаях $(n=167)$ оценены размеры $\Lambda \mathrm{B}$ и $\Lambda$ по данным MCKT, а также варианты их впадения в $\Lambda$ П. При описании кАинической характеристики пациентов в 146 случаях $(87,4 \%)$ учтена форма ФП, наличие артериальной гипертонии (АГ), сахарного диабета (СД) и мегочной гипертензии $(\Lambda \Gamma)$. У 21 пациента (12,6\%) данный анализ не выполнен в связи с отсутствием уточненных клинических данных, а также информации, позволяющей разделить пациентов на группы с пароксизмальной и персистирующей формами ФП (табл. №1).
Протокол выполнения мультиспиральной компьютерной томографии.

Bce пациенты подписывали информированное согласие на проведение исследования. Мультиспиральная компьютерная томография (MCKT) выполнялась на 256-срезовом и 64срезовом компьютерных томографах (Philips Brilliance iCT/Philips Brilliance 64, Германия). Сканирование проводимось при задержке пациентом дыхания на вдохе. Область сканирования - от бифуркации трахеи до верхушки сердца. Исследование проводимось синхронизировано с регистрацией электрокардиограммы, сканирование выполнялось на 40\% фазе интервала RR. Использовались следующие параметры сканирования: комиимация 2x128x0,625 мм /64x0,625, напряжение на трубке 100 кВ, экспозиция 150 мАс. Исследование проводилось с внутривенным болюсным введением 80 м неионного контрастного вещества (УАьтравист-370, Вayer, Германия/Йомерон-400, Вracco, Италия) со скоростью 4-5 м $/$ с, с последующим введением 50 м физиологического раствора. Запуск сканирования осуществлялся по триггеру болюса, порог плотности на восходящей аорте 110 ед. Н, послепороговая задержка минимальная.

Протокол анализа данных мультиспиральной компьютерной томографии.

Постпроцессорная обработка изображений с толщиной среза 1,0 мм и интервалом реконструкции 0,6 мм проводимась на рабочей станции Brilliance Workspace Portal, Германия. Применялись мультипланарные реконструкции в аксиальной, корональной, сагиттальной, косых проекциях. Выполнялись 3Dреконструкции дмя определения анатомических вариантов мегочных вен $(\Lambda \mathrm{B})$. Устья $\Lambda \mathrm{B}$ опреде-

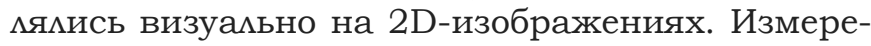
ния диаметра устьев $\Lambda$ В проводими перпендикулярно устью с использованием реконструкции вдоль оси $\Lambda \mathrm{B}$. При дмине ствола $\Lambda \mathrm{B}$, не превышающей значение медианы, последние считали "короткими". Если диина ствола $\Lambda \mathrm{B}$ превышала значение медианы данного показателя, то данные стволы $\Lambda \mathrm{B}$ при анализе расцениваАись как "длинные".

При описании вариантов впадения мегочных вен $(\Lambda \mathrm{B})$ в мевое предсердие $(\Lambda П)$ учитывамись следующие признаки:

1. Комичество раздемьных ипсилатеральных устьев $\Lambda \mathrm{B}$;

2. Слияние ипсилатеральных $\Lambda \mathrm{B}$ в один ство до впадения в $\Lambda$ с формированием коц$\Lambda$ ектора $\Lambda$;

3. Наличие более четырех раздемьных устьев $\Lambda \mathrm{B}$, впадающих в $\Lambda П$.

4. Слияние контрлатеральных $\Lambda \mathrm{B}$ в один ство до впадения в $\Lambda$ с формированием коц$\Lambda$ ектора $\Lambda \mathrm{B}$; 
Табинца №1. Характернстика пациентов.

\begin{tabular}{|c|c|c|}
\hline Параметры & $\mathbf{n}$ & $\%$ \\
\hline \multicolumn{3}{|l|}{ Масса тела } \\
\hline Дефицит массы тела & 1 & 0,6 \\
\hline Нормальная масса тела & 22 & 13,2 \\
\hline Избыточная масса тела & 64 & 38,3 \\
\hline Ожирение & 80 & 47,9 \\
\hline \multicolumn{3}{|l|}{ Форма фибрилляции предсердий } \\
\hline Пароксизмальная & 107 & 64,1 \\
\hline Персистирующая & 39 & 23,3 \\
\hline Разделение на пароксизмальную и персистирующую формы не проведено & 21 & 12,6 \\
\hline \multicolumn{3}{|l|}{ Сопутствующие заболевания } \\
\hline Артериальная гипертония & 107 & 64,1 \\
\hline Сахарный диабет 2 типа & 15 & 9,0 \\
\hline Хроническая сердечная недостаточность & 42 & 25,1 \\
\hline Легочная гипертензия & 5 & 3,0 \\
\hline
\end{tabular}

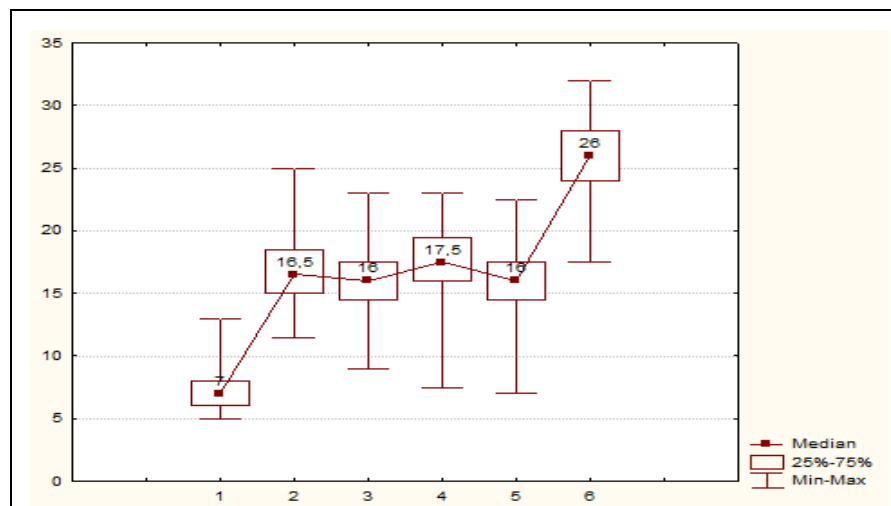

Pис. 1 (Fig. 1)

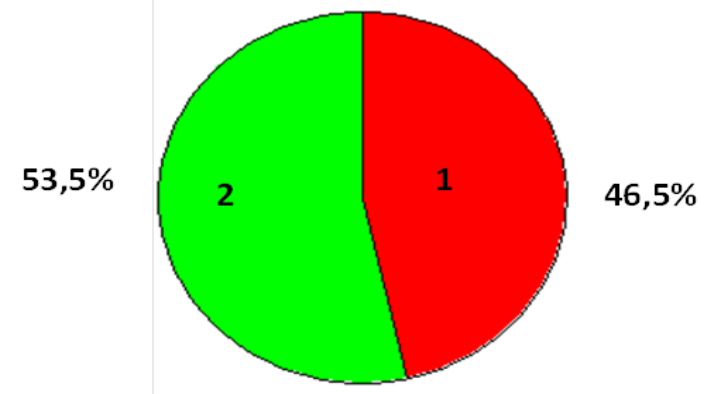

Puc. 2 (Fig. 2)
Рис. 1. Аиаметр разАельных устьев и комлекторов ^егочных вен $(\Lambda B)$.

1 - добавочная $\Lambda \mathrm{B} ; 2$ - мевая верхняя $\Lambda \mathrm{B} ; 3$ - мевая нижняя $\Lambda \mathrm{B} ; 4$ - правая верхняя $\Lambda \mathrm{B} ; 5$ - правая нижняя $\Lambda \mathrm{B} ; 6$ - комлектор $\Lambda \mathrm{B}$.

Fig. 1. Values of the separate and common pulmonary vein (PV) ostia diameters.

1 - Accessory PV; 2 - Left superior PV; 3 - Left inferior PV; 4 - Right superior PV; 5 - Right inferior PV; 6 Common PV ostium.

\section{5. Сочетание признаков.}

Статистический анализ.

Количественные признаки представцены в виде Min - Max (Me, IQR), где Me - медиана, a IQR (25\%Q-75\%Q) - интерквартильный размах. При статистических расчетах применялись непараметрические критерии. Расчет статистической значимости размичии непрерывных признаков проводимся по методу Манна-Уитни. Дия проверки гипотез о независимости номинамьных признаков использовались таблицы сопря-

\section{Рис. 2. Аиаграмма.}

Частота встречаемости длинных и коротких стволов комлекторов мегочных $(\Lambda \mathrm{B})(\mathrm{n}=43)$.

Примечание:

1 - короткий ствол $\Lambda$ В (диина менее 16 мм);

$2-$ длинный ство $\Lambda$ В (длина = или > 16 мм).

Fig. 2. Diagram.

The prevalence of long and short trunks of the pulmonary veins (PV) with common ostium.

1 - short PV trunk (length $<16 \mathrm{~mm}$ );

$2-$ long PV trunk (length $=$ or $>16 \mathrm{~mm}$ ).

женности с оценкой критерия Хи-квадрат Пирсона (х2). Дия исследования симы связи между номинальными признаками использовался коэффициент Cramer's V. При проведении множественных сравнений применялся непараметрический дисперсионный анализ с использованием критерия Краскела-Уомлиса (далее Kruskal-Wallis test). При проверке статистических гипотез принимался 5\% уровень значимости. Нулевая гипотеза об отсутствии различий отклонялась, если вероятность ошибочно ее от- 


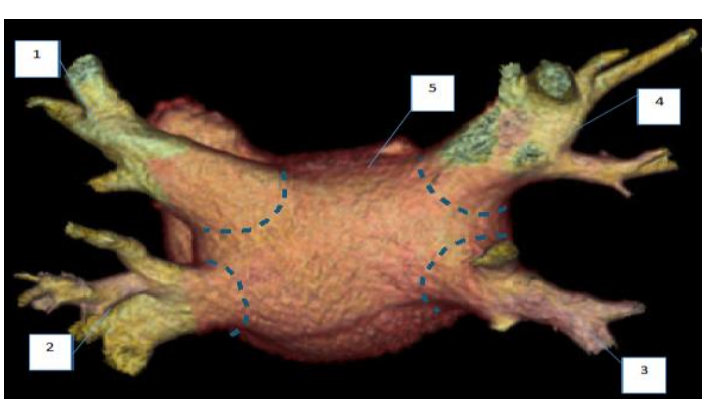

Рис. 3 (Fig. 3)

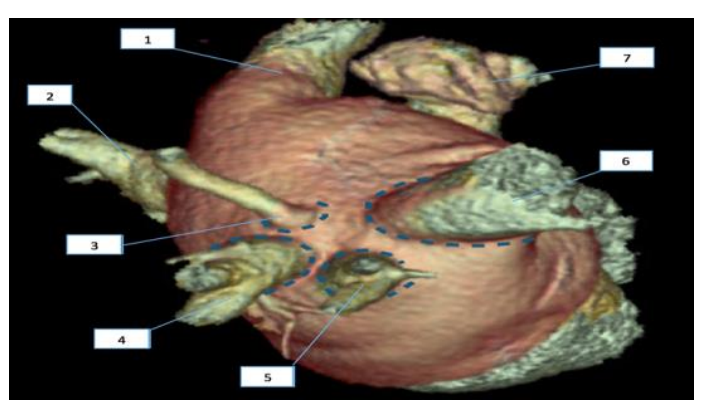

Рис. 4 (Fig. 4)

\section{Рис. 3. 3D-модель.}

Типичная анатомия (Вариант 1): отмечается наличие четыpeх раздельных устьев мегочных вен $(\Lambda \mathrm{B})(\mathrm{n}=88 ; 52,7 \%) .1$ $\Lambda$ евая верхняя $\Lambda \mathrm{B} ; 2-$ мевая нижняя $\Lambda \mathrm{B} ; 3$ - правая нижняя $\Lambda \mathrm{B} ; 4$ - правая верхняя $\Lambda \mathrm{B} ; 5$ - задняя стенка мевого предсердия. Вид сзади. Пунктирной минией обозначены устья $\Lambda \mathrm{B}$.

\section{Fig. 3. 3D-model.}

Normal anatomy (Type 1) $(\mathrm{n}=88 ; 52.7 \%)$ : four independent pulmonary veins with four separate ostia.1 - Left superior pulmonary vein (PV); 2 - Left inferior PV; 3 - Right inferior PV; 4 - Right superior PV; 5 - Left atrium posterior wall. Posterior view. The dotted lines indicate the PV ostia.

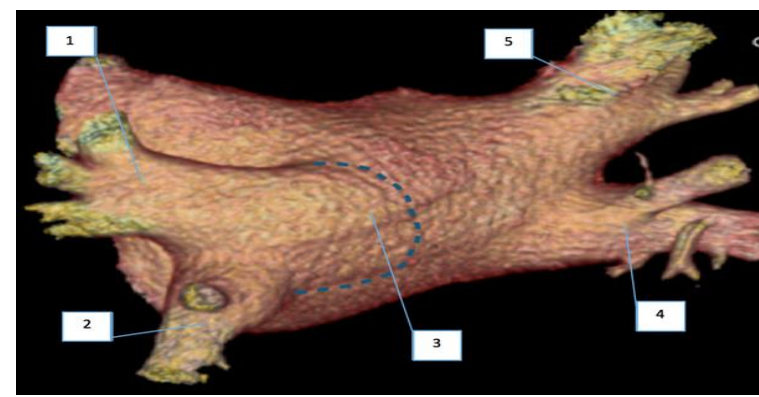

Рис. 5 (Fig. 5)

Рис. 5. 3D-моАень.

Комлектор мегочных вен $(\Lambda \mathrm{B})$ слева (Вариант 3): цевая верхняя и нижняя $\Lambda \mathrm{B}$ сливаются в один ствоц до впадения в мевое предсердие ( $\Lambda$ П), (n=31; 18,6\%). $1-\Lambda$ евая верхняя $\Lambda \mathrm{B}$; 2 - мевая нижняя $\Lambda \mathrm{B} ; 3$ - ство мевых $\Lambda \mathrm{B} ; 4$ - правая нижняя $\Lambda \mathrm{B} ; 5-$ правая верхняя $\Lambda$ В.Вид сзади слева. Пунктир-

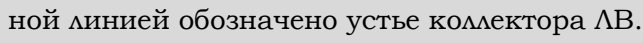

\section{Fig. 5. 3D-model.}

Left common pulmonary vein (PV) ostium (Type 3): coalescence of the left superior and left inferior PVs proximal to the left atrium ( $\mathrm{n}=31 ; 18.6 \%) .1$ - Left superior PV; 2 - Left inferior PV; 3 - Left PV trunk; 4 - Right inferior PV; 5 - Right superior PV.Left posterior oblique view. The dotted lines indicate the common PV ostium
Рис. 4. 3D-молель.

Рассыпной тип (Вариант 2): наличие трех или более раздельных ипсилатеральных устьев мегочных вен $(\Lambda \mathrm{B})$. В дан-

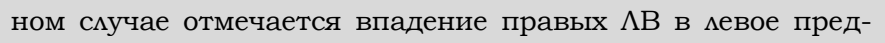
сердие $(\Lambda П)$ четырьмя раздельными устьями (n=36; 21,5\%). 1 - Аевая верхняя $\Lambda \mathrm{B} ; 2$ - мевая нижняя $\Lambda \mathrm{B} ; 3,5$ - добавочные $\Lambda \mathrm{B}$ справа; 4 - правая нижняя $\Lambda \mathrm{B} ; 6$ - правая верхняя $\Lambda \mathrm{B} ; 7$

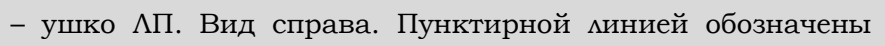
устья $\Lambda$ В справа.

\section{Fig. 4. 3D-model.}

Dichotomous pattern (Type 2): three or more independent ipsilateral pulmonary vein (PV) ostia. Right PVs have four independent ostia in this case $(\mathrm{n}=36 ; 21.5 \%) .1$ - Left superior PV; 2 - Left inferior PV; 3, 5 - right accessory PVs; 4 - Right inferior PV; 6 - Right superior PV; 7 - Left atrial appendage; Right lateral view. The dotted lines indicate the right PV ostia.

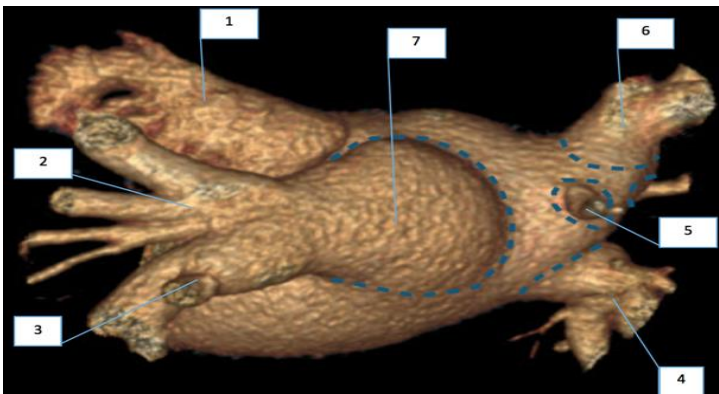

Рис. 6 (Fig. 6)

\section{Рис. 6. 3D-молель.}

Смешанный тип (Вариант 4): сочетание комектора мегочных вен $(\Lambda \mathrm{B})$ с рассыпным типом строения $(\mathrm{n}=10 ; 6 \%)$. В данном случае отмечается наличие комлектора $\Lambda \mathrm{B}$ слева и рассыпного типа справа.1 - Ушко мевого предсердия; 2 мевая верхняя $\Lambda \mathrm{B} ; 3-$ мевая нижняя $\Lambda \mathrm{B} ; 4-$ правая нижняя $\Lambda \mathrm{B} ; 5$ - добавочная правая $\Lambda \mathrm{B} ; 6$ - правая верхняя $\Lambda \mathrm{B} ; 7$ -

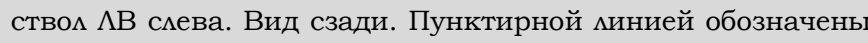
устья $\Lambda \mathrm{B}$.

\section{Fig. 6. 3D-model.}

Combined pattern (Type 4): common pulmonary vein (PV) ostium and dichotomous pattern combination $(n=10 ; 6 \%)$. Thera are a common left PV ostium and right dichotomous pattern in this case. 1 - Left atrial appendage; 2 - Left superior PV; 3 Left inferior PV; 4 - Right inferior PV; 5 - Right accessory PV; 6 - Right superior PV; 7 - Left PV trunk.Posterior view. The dotted lines indicate the PV ostia. 


\section{RUSSIAN ELECTRONIC JOURNAL OF RADIOLOGY}

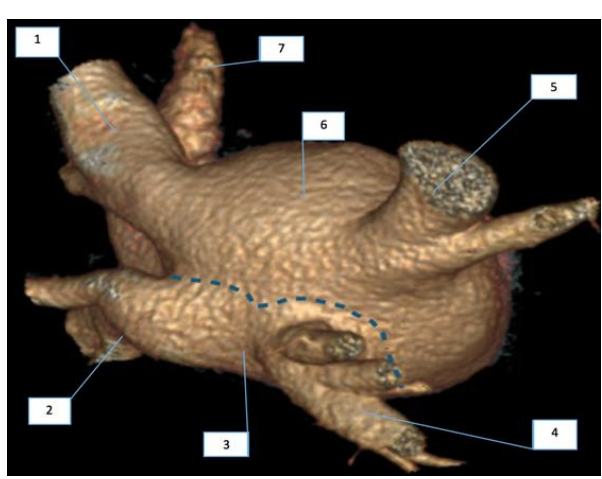

Рис. 7 (Fig. 7)

\section{Рис. 7. 3D-моАень.}

Комлектор контрлатеральных мегочных вен $(\Lambda \mathrm{B})$ (Вариант 5): слияние контрлатеральных $\Lambda \mathrm{B}$ в один ствол до впадения в мевое предсердие $(\Lambda П)(\mathrm{n}=1 ; 0,6 \%) .1-\Lambda$ евая верхняя $\Lambda \mathrm{B} ; 2$ мевая нижняя $\Lambda \mathrm{B} ; 3$ - общий ство контрлатеральных $\Lambda \mathrm{B} ; 4$ - правая нижняя мегочная вена; 5 - правая верхняя $\Lambda \mathrm{B} ; 6$ крыша $\Lambda П ; 7$ - ушко $\Lambda$. Вид сзади справа. Пунктирной минией обозначено общее устье нижних правой и мевой $\Lambda \mathrm{B}$. Fig. 7. 3D-model.

Contralateral pulmonary vein (PV) common ostium (Type 5): coalescence of the right and left inferior PVs proximal to the left atrium (LA) ( $\mathrm{n}=1 ; 0.6 \%) .1$ - Left superior PV; 2 - Left inferior PV; 3 - Contralateral PV common trunk; 4 - Right inferior PV; 5 - Right superior PV; 6 - LA roof; 7 - LA appendage. Right posterior oblique view. The dotted lines indicate the right and left common PV ostium.

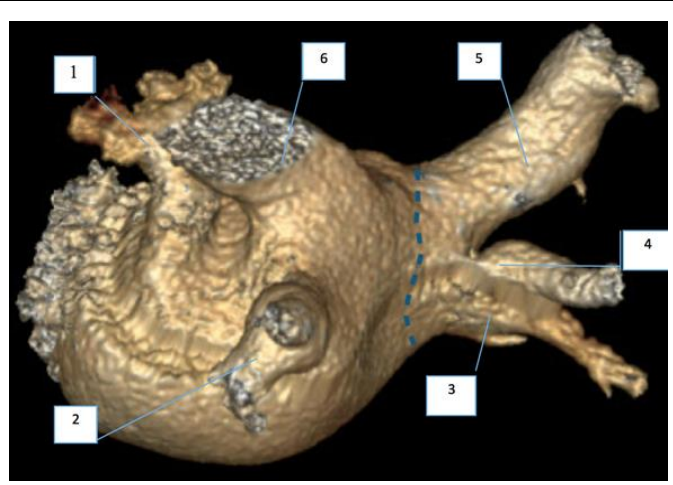

Рис. 8 (Fig. 8)

Комектор мегочных вен $(\Lambda \mathrm{B})$ справа (Вариант 6):правые $\Lambda \mathrm{B}$ сливаются с общий ствол до впадения в мевое предсердие $(\Lambda П)(\mathrm{n}=1 ; 0,6 \%) .1$ - Ушко мевого предсердия; 2 - мевая нижняя $\Lambda \mathrm{B} ; 3$ - правая нижняя $\Lambda \mathrm{B} ; 4$ - добавочная правая $\Lambda \mathrm{B} ; 5$ - правая верхняя $\Lambda \mathrm{B} ; 6$ - мевая верхняя $\Lambda \mathrm{B}$. Вид сзади слева. Пунктирной минией обозначено устье комлектора $\Lambda \mathrm{B}$ справа.

\section{Fig. 8. 3D-model.}

Right common pulmonary vein (PV) ostium: coalescence of the right PVs proximal to the left atrium (LA) (Type 6): $(n=1 ; 0.6 \%)$.

1 - LA appendage; 2 -Left inferior PV; 3 - Right inferior PV; 4 Right accessory PV; 5 - Right superior PV; 6 - Left superior PV.

Left posterior oblique view. The dotted lines indicate the common ostium of the right PVs.

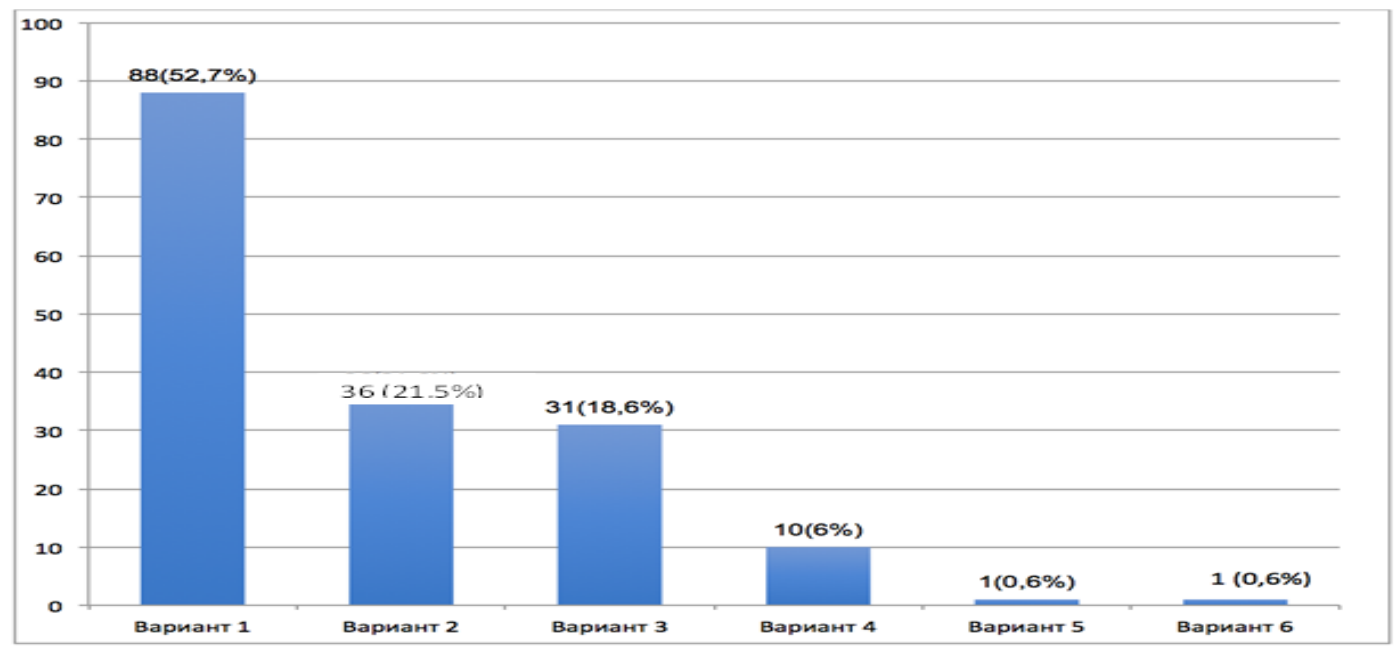

Pис. 9 (Fig. 9)

\section{Рис. 9. Аиаграмма.}

Варианты дистальной анатомии мегочных $(\Lambda \mathrm{B})$ : Вариант 1 - типичная анатомия; Вариант 2 - рассыпной тип; Вариант 3 комлектор мевых $\Lambda$ В; Вариант 4 - смешанный тип; Вариант 5 - комлектор контрлатеральных $\Lambda$ В; Вариант 6 - комлектор правых $\Lambda \mathrm{B}$.

\section{Fig. 9. Diagram.}

Anatomical variations in pulmonary veins (PV):Type 1 - Normal anatomy; Type 2 - Dichotomous type; Type 3 - Left common PV ostium; Type 4 - Combined pattern; Type 5 - Contralateral PV common ostium; Type 6 - Right common PV ostium. 
вергнуть не превышала 5\%.

Результаты.

При анализе данных МСКТ выявлена значительная вариабельность диаметров устьев пёгочных вен $(\Lambda \mathrm{B})$ при их раздемьном впадении в $\Lambda$ П. Диаметр устья мевой верхней $\Lambda$ В варьировал в диапазоне 11,5-25 (Me=17, IQR: 15,5-19) Mм $(n=125)$. Диаметр мевой нижней $\Lambda \mathrm{B}$ состави 9-23 (Me=16, IQR: 14,7-18) мм (n=125). Правая верхняя $\Lambda \mathrm{B}$ принимала значения 7,5-23 (Me=17,5, IQR: 16-19,5) мм (n=165). Правая нижняя $\Lambda \mathrm{B}-7-22,5$ (Me=16, IQR: $14,8-17,5) \mathrm{Mм}$ $(n=165)$. Выявцено, что диаметр устьев добавочных $\Lambda$ в колебался в диапазоне 5-13 (Me=7, IQR: 6-8) мм (n=56) (рис. 1).

У 43 пациентов $(25,7 \%)$ отмечено образование комлектора $\Lambda$ В до впадения в $\Lambda$. Диина ствола комлектора $\Lambda$ В составима 7-28 (Mе=16, IQR: 11-20) мм. Короткие (дмина менее 16 мм) ствоцы комлектора $\Lambda$ В выявцены в 20 из 43 случаев (46,5\%), дминные - у 23 из 43 больных (53,5\%) (рис. 2).

Выявлены следующие типы комлекторов $\Lambda \mathrm{B}(\mathrm{n}=43)$ : комлектор $\Lambda \mathrm{B}$ слева $(\mathrm{n}=41 ; 95,4 \%)$, комлектор правых $\Lambda$ B $(\mathrm{n}=1 ; 2,3 \%)$, комлектор контрлатерамьных $\Lambda \mathrm{B}(\mathrm{n}=1 ; 2,3 \%)$. Диаметр ко $\Lambda$ екторов $\Lambda$ В вен варьировал в диапазоне 17,532 (Ме=26, IQR: 24-27) мм (рис. 1). $\Lambda \mathrm{B}$ в $\Lambda \Pi$ :

Выявцены следующие варианты впадения

1. Типичная анатомия (рис. 3): наличие четырех раздемьных устьев $\Lambda \mathrm{B}(\mathrm{n}=88 ; 52,7 \%)$.

2. Рассыпной тип (рис. 4): наличие трех и более ипсиматеральных устьев $\Lambda \mathrm{B}(\mathrm{n}=36 ; 21,5 \%)$.

3. Комлектор мевых $\Lambda \mathrm{B}$ (рис. 5): мевая верхняя и нижняя $\Lambda$ В сливаются в один ство до впадения в $\Lambda$, правые мегочные вены впадают в $\Lambda$ двумя раздемьными устьями $(\mathrm{n}=31$; $18,6 \%)$.

4. Смешанный тип (рис. 6): сочетание коммектора $\Lambda \mathrm{B}$ с одной стороны и рассыпного типа с другой (n=10; 6\%).

5. Комлектор контрлатеральных $\Lambda$ В (рис. 7): слияние контрцатеральных $\Lambda$ В в один ство до впадения в $\Lambda$ ( $\mathrm{n}=1 ; 0,6 \%)$.

6. Комлектор правых $\Lambda \mathrm{B}$ (рис. 8) (n=1; 0,6\%): Правые $\Lambda$ В сливаются в общий ство до

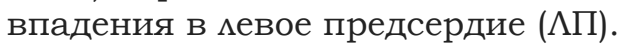

Выявлено, что при наличии у пациентов фибримляции предсердий (ФП) типичное впадение $\Lambda$ В в $\Lambda$ (Вариант 1) встречалось в $52,7 \%$ случаев. Рассыпной тип (Вариант 2) - реже (21,5\%). С иияние мевых $\Lambda$ В в один ство до впадения в $\Lambda$ (Вариант 3 ) обнаружено у 18,6\% обследованных. Сочетание комлектора $\Lambda \mathrm{B}$ с наличием рассыпного типа составимо 6\%. Комлектор контрлатеральных $\Lambda$ В (Вариант 5) и комлектор $\Lambda \mathrm{B}$ справа (Вариант 6) явицись наиболее редкими вариантами впадения $\Lambda \mathrm{B}$ в $\Lambda$. Суммарная частота встречаемости этих анатомических паттернов не превысила 1,2\% (рис. 9).

\section{Обсуждение.}

Типичная анатомия дистального отдела мёгочных вен $(\Lambda \mathrm{B})$ у пациентов без анамнестических указаний на фибримляцию предсердий (ФП) быма детально изучена в серии ранее проведенных исследований. Анализируя эти анатомические особенности у пациентов без ФП по данным аутопсий $(n=130)$, Klimek-Piotrowska W. et al. выдемили три основных анатомических паттерна: типичная анатомия, мевый общий ство $\Lambda$ В и наличие добавочной $\Lambda$ В справа. Авторы обнаружили, что частота встречаемости типичного впадения $\Lambda \mathrm{B}$ в $\Lambda$ состав $я е$ т $71 \%$ [19]. В нашей работе типичный вариант впадения $\Lambda$ в в $\Lambda$ отмечался в $52,7 \%$ случаев. Разцичия в частоте встречаемости анатомических вариантов $\Lambda$ В при ФП, по сравнению с таковыми у пациентов без данной тахиаритмии, объясняет интерес исследователей к данному вопросу [20].

Изучая вариационную анатомию $\Lambda \mathrm{B}$ перед их катетерной изоляцией, Kato R. et al. описали 6 типов впадения $\Lambda \mathrm{B}$ в $\Lambda$ : "типичная анатомия", "короткий общий мевый ствоц", "диинный общий $е$ евый ство "; "средняя правая $\Lambda$ В"; "две средних $\Lambda$ ", "средняя правая $\Lambda$ и и верхняя правая добавочная $\Lambda$ В". Смешанный тип (Вариант 4), комлектор правых $\Lambda$ В (Вариант 6) и комектор контрлатеральных $\Lambda$ В (Вариант 5) в исследовании Kato R. et al. не выделяется, что может быть обусловлено как меньшим (n=28) по сравнению с нашим $(\mathrm{n}=167)$ количеством наблюдений, так и акцентом авторов на размичные мокализации впадения сегментарных вен [21]. Могическим продомжением этой работы стало исследование Marom E et. al., в которой описание анатомии $\Lambda \mathrm{B}$ основано на подсчете количества устьев $\Lambda \mathrm{B}$, впадающих в $\Lambda$, наличия сегментарных вен, а также удаменность их от места впадения в основную $\Lambda \mathrm{B}$ (n=201). Авторы выдемяют 10 паттернов $\Lambda \mathrm{B}$ справа и 4 слева [22]. Недостатком данной классификации является избыточное количество типов впадения $\Lambda \mathrm{B}$ в $\Lambda \Pi$, затрудняющее ее практическое использование и не учитывающее редкие анатомические вариации, описанные в настоящей работе. Так, в нашем исследовании приведен крайне редко встречающийся вариант впадения $\Lambda \mathrm{B}$ в $\Lambda$, ранее опубликованный Defaye P. et al. в качестве клинического наблюдения. Как и в нашем исследовании, авторы описали случай слияния контрлатеральных $\Lambda \mathrm{B}$ в общий ствоц, также отмечая крайне редкую встречаемость данной анатомической особенности [23].

Наиболее близкое к нашей классификации описание анатомических вариантов $\Lambda \mathrm{B}$ приве Bittner A. et al. Авторы описывают 6 анатомических вариантов, выдемяя при этом "короткий» 
и "диинный" ство евых $\Lambda$ В [20]. Как и в нашем исследовании, авторы описывают комлектор правых $\Lambda \mathrm{B}$, также указывая на крайне редкую частоту встречаемости данного анатомического варианта. Раздемение комлектора мевых $\Lambda \mathrm{B}$ на два подтипа "короткий" и "дминный" ство в нашем исследовании не проводилось, в связи с тем, что в настоящее время убедительные доказательства о влиянии этого фактора на течение ФП, а также резумьтаты катетерной абляции в митературе не представмены. Наличие рассыпного типа строения $\Lambda \mathrm{B}$, приведенного в нашем исследовании, Bittner A. et al. не выделяют, от-

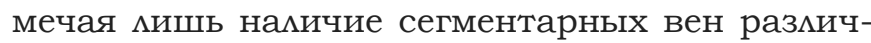
ной мокализации. При этом, наличие дополнительных сегментарных $\Lambda \mathrm{B}$ вне зависимости от их места впадения в $\Lambda$ может быть ассоциировано с повышением продомжительности воздействия при КИАВ [24, 25]. В нашем исследовании случаи наличия сегментарных вен объединены в рассыпной тип впадения $\Lambda \mathrm{B}$ (Вариант 2) в связи с тем, что наличие более четырех устьев мегочных вен может быть взаимосвязано с результатом катетерной абляции.

\section{Cписок митературы:}

1. Wilke, T., Groth, A., Mueller, S., Pfannkuche, M., Verheyen, F., Linder, et al. Incidence and prevalence of atrial fibrillation: an analysis based on 8.3 million patients. Europace. 2013; 15 (4): 486-493. doi: 10.1093/europace/eus333. Epub 2012 Dec 6.

2. Camm, A. J., Lip, G. Y., De Caterina, R., Savelieva, I., Atar, D., Hohnloser, S. H., et al. ESC Committee for Practice Guidelines (CPG). 2012 focused update of the ESC Guidelines for the management of atrial fibrillation: an update of the 2010 ESC Guidelines for the management of atrial fibrillation. Developed with the special contribution of the European Heart Rhythm Association. European Heart Journal. 2010; 33 (21): 2719-2747.

3. Calkins H., Kuck K.H., Cappato R., Brugada J, Camm A.J., Chen S.A., et al. 2012 HRS/EHRA/ECAS expert consensus statement on catheter and surgical ablation of atrial fibrillation: recommendations for patient selection, procedural techniques, patient management and follow-up, definitions, endpoints, and research trial design. Heart Rhythm. 2012; 9 (4): 632-696. doi: 10.1007/s10840-012-9672-7.

4. Bunch, T. J., \& Cutler, M. J. Is pulmonary vein isolation still the cornerstone in atrial fibrillation ablation. Journal of thoracic disease. 2015; 7 (2): 132. doi: 10.3978/j.issn.2072 1439.2014.12.46.

5. Gaita, F., Scaglione, M., Battaglia, A., Matta, M., Gallo, C., Galatà, M., et al. Very long-term outcome following transcatheter ablation of atrial fibrillation. Are results maintained after 10 years of follow up. Ep Europace. 2017; 20 (3): 443-450. doi: 10.21037/jtd.2017.09.128.

6. Sohns, C., Sohns, J. M., Bergau, L., Sossalla, S., Vollmann, D., Lüthje, L., et al. Pulmonary vein anatomy predicts freedom from atrial fibrillation using remote magnetic navigation for circumferential pulmonary vein ablation. Europace. 2013; 15 (8): 1136-1142. doi: 10.1093/europace/eut059.

7. Güler, E., Güler G. B., Demir G. G., Kizilirmak F., Güneş H. M., Barutcu I. et al. Effect of pulmonary vein anatomy and pulmo-

\section{Заключение.}

Дистальная анатомия мегочных вен характеризуется выраженной вариабельностью. У больных с ФП типичный анатомический паттерн впадения $\Lambda \mathrm{B}$ в $\Lambda$ встречается в $52,7 \%$ случаев. Реже встречаются рассыпной тип строения $\Lambda \mathrm{B}$, наличие комлектора $\Lambda \mathrm{B}$, а также их сочетание. Комлекторы правых $\Lambda \mathrm{B}$ и контрматеральных $\Lambda$ В являются крайне редкими анатомическими вариантами. Выдемение шести вариантов впадения $\Lambda \mathrm{B}$ в $\Lambda П$ (Вариант 1 - типичная анатомия; Вариант 2 - рассыпной тип; Вариант 3 - комлектор мевых $\Lambda$ В; Вариант 4 смешанный тип; Вариант 5 - комлектор контрматеральных $\Lambda$ В; Вариант 6 - комлектор правых $\Lambda B)$ позволяет описать особенности дистальной анатомии мегочных вен по данным MCKT.

\section{интересов.}

Источник финансирования и конфликт

Авторы данной статьи подтвердими отсутствие финансовой поддержки исследования и конфмикта интересов, о которых необходимо сообщить.

nary vein diameters on outcome of cryoballoon catheter ablation for atrial fibrillation. Pacing and Clinical Electrophysiology. 2015; 38 (8): 989-996. doi: 10.1111/pace. 12660.

8. Koźluk, E., Zyśko, D., Piatkowska, A., Kiliszek, M., Lodziński, P., Malkowska, S., et al. Effectiveness comparison of various atrial fibrillation ablation methods in patients with common venous trunk. Advances in clinical and experimental medicine: official organ Wroclaw Medical University. 2018.

9. Shi, Y., Mi, S., Shi, Y., Wang, H., Li, J., Yu, B., et al. Evaluation of pulmonary vein anatomy using 256-slice computed tomography. Turkish journal of medical sciences. 2017; 47 (5): 1526-1534. doi: 10.2174/157340309787048121.

10. Odozynski, G., Forno, A. R. J. D., Lewandowski, A., Nascimento, H. G., \& d'Avila A. Paroxysmal Atrial Fibrillation Catheter Ablation Outcome Depends on Pulmonary Veins Anatomy. Arquivos brasileiros de cardiologia, (AHEAD). 2018. doi: 10.5935/abc.20180069.

11. Haissaguerre, M., Jaïs, P., Shah, D. C., Takahashi, A., Hocini, M., Quiniou, G., et al. Spontaneous initiation of atrial fibrillation by ectopic beats originating in the pulmonary veins. New England Journal of Medicine. 1998; 339 (10): 659-666. doi: 10.1056/NEJM199809033391003.

12. Стеклов В. И., Морозов Д. А., Емельяненко М. В., Кузьменков Д. В. Псевдоротаиионная атриография. Российский электронный журнал лучевой диагностики 2015; 5 (1): 16 18.

13. Каштанова Н.Ю., Груздев И.С., Кондратьев Е.В., Артюхина Е.А., Яшков М.В., Ревишвили А.Ш. Мультиспиральная компьютерная томография сердиа: оптимизаиия протокола сканирования при проведении неинвазивного картирования сердиа перед катетерной абляиией фибрилляиии предсердий. Медииинская визуализаиия. 2018; 3: 33-48. https://doi.org/ 10.24835/1607-0763-2018-3-33-48

14. Kiuchi, K., Yoshida, A., Takei, A., Fukuzawa, K., Itoh, M., 
Imamura, K., et al. Topographic variability of the left atrium and pulmonary veins assessed by 3D-CT predicts the recurrence of atrial fibrillation after catheter ablation. Journal of arrhythmia. 2015; 31 (5): 286-292. doi: 10.1016/j.joa.2015.03.006.

15. Jongbloed, M. R., Dirksen, M. S., Bax, J. J., Boersma, E., Geleijns, K., Lamb, H. J., et al. Atrial fibrillation: multi-detector row CT of pulmonary vein anatomy prior to radiofrequency catheter ablation-initial experience. Radiology. 2005; 234 (3): 702 709. doi: 10.1148/ radiol.2343031047.

16. Kanaji, Y., Miyazaki, S., Iwasawa, J., Ichihara, N., Takagi, T., Kuroi, A., et al. Pre-procedural evaluation of the left atrial anatomy in patients referred for catheter ablation of atrial fibrillation. Journal of cardiology. 2016; 67 (1): 115-121. doi: 10.1016/j.jjcc.2015.02.016.

17. Thorning, C., Hamady, M., Liaw, J. V. P., Juli, C., Lim, P. B., Dhawan, R., et al. CT evaluation of pulmonary venous anatomy variation in patients undergoing catheter ablation for atrial fibrillation. Clinical imaging. 2011; 35 (1): 1-9. doi: 10.1371/journal.pcbi.1006166.

18. Chen, J., Yang, Z. G., Xu, H. Y., Shi, K., Long, Q. H., \& Guo, $Y$. K. Assessments of pulmonary vein and left atrial anatomical variants in atrial fibrillation patients for catheter ablation with cardiac CT. European radiology. 2017; 27 (2): 660-670. doi: 10.1007/s00330-016-4411-6.

19. Klimek-Piotrowska, W., Hołda, M. K., Piatek, K., Koziej, M., \& Hołda, J. Normal distal pulmonary vein anatomy. 2016; 4 : 1579. doi: 10.7717/peerj. 1579.

20. Bittner, A., Mönnig, G., Vagt, A. J., Zellerhoff, S., Wasmer,

\section{References:}

1. Wilke, T., Groth, A., Mueller, S., Pfannkuche, M., Verheyen, F., Linder, et al. Incidence and prevalence of atrial fibrillation: an analysis based on 8.3 million patients. Europace. 2013; 15(4): 486-493. doi: 10.1093/europace/eus333. Epub 2012 Dec 6.

2. Camm, A. J., Lip, G. Y., De Caterina, R., Savelieva, I., Atar, D., Hohnloser, S. H., et al. ESC Committee for Practice Guidelines (CPG). 2012 focused update of the ESC Guidelines for the management of atrial fibrillation: an update of the 2010 ESC Guidelines for the management of atrial fibrillation. Developed with the special contribution of the European Heart Rhythm Association. European Heart Journal, 2010; 33 (21): 2719-2747.

3. Calkins H., Kuck K.H., Cappato R., Brugada J, Camm A.J., Chen S.A., et al. 2012 HRS/EHRA/ECAS expert consensus statement on catheter and surgical ablation of atrial fibrillation: recommendations for patient selection, procedural techniques, patient management and follow-up, definitions, endpoints, and research trial design. Heart Rhythm. 2012; 9 (4): 632-696. doi: 10.1007/s10840-012-9672-7.

4. Bunch, T. J., \& Cutler, M. J. Is pulmonary vein isolation still the cornerstone in atrial fibrillation ablation. Journal of thoracic disease. 2015; 7 (2): 132. doi: 10.3978/j.issn.20721439.2014.12.46.

5. Gaita, F., Scaglione, M., Battaglia, A., Matta, M., Gallo, C., Galatà, M., et al. Very long-term outcome following transcatheter ablation of atrial fibrillation. Are results maintained after 10 years of follow up. Ep Europace. 2017; 20 (3): 443-450. doi: 10.21037/jtd.2017.09.128.

6. Sohns, C., Sohns, J. M., Bergau, L., Sossalla, S., Vollmann, D., Lüthje, L., et al. Pulmonary vein anatomy predicts freedom from atrial fibrillation using remote magnetic navigation for cir-
K., Köbe, J., et al. Pulmonary vein variants predispose to atrial fibrillation: a case-control study using multislice contrastenhanced computed tomography. Europace. 2011; 13 (10): 1394-1400. doi: 10.1093/europace/eur145.

21. Kato, R., Lickfett, L., Meininger, G., Dickfeld, T., Wu, R., Juang, G., et al. Pulmonary vein anatomy in patients undergoing catheter ablation of atrial fibrillation: lessons learned by use of magnetic resonance imaging. Circulation. 2003; 107 (15): 2004 2010. doi: 10.1161/01.CIR.0000061951.81767.4E.

22. Marom, E. M., Herndon, J. E., Kim, Y. H., \& McAdams, H. P. Variations in pulmonary venous drainage to the left atrium: implications for radiofrequency ablation. Radiology. 2004; 230 (3): 824-829. doi: 10.1148/radiol.2303030315.

23. Defaye, P., Kane, A., \& Jacon, P. An unusual connection of the right and left inferior pulmonary veins in the left atrium via a common ostium. Heart. 2010; 96 (23): 1951. doi: 10.1136/ha.2010.002899.

24. McLellan, A. J., Ling, L. H., Ruggiero, D., Wong, M. C., Walters, T. E., Nisbet, A., et al. Pulmonary vein isolation: the impact of pulmonary venous anatomy on long-term outcome of catheter ablation for paroxysmal atrial fibrillation. Heart Rhythm. 2014; 11 (4): 549-556. doi: 10.1016/j.hrthm.2013.12.025.

25. Wei, H. Q., Guo, X. G., Zhou, G. B., Sun, Q., Yang, J. D., Xie, $H$. Y., et al. Procedural findings and clinical outcome of second-generation cryoballoon ablation in patients with variant pulmonary vein anatomy. Journal of cardiovascular electrophysiology. 2018.

cumferential pulmonary vein ablation. Europace. 2013; 15 (8): 1136-1142. doi: 10.1093/europace/eut059.

7. Güler, E., Güler, G. B., Demir, G. G., Kizilirmak, F., Güneş, H. M., Barutcu, $\dot{I}$., et al. Effect of pulmonary vein anatomy and pulmonary vein diameters on outcome of cryoballoon catheter ablation for atrial fibrillation. Pacing and Clinical Electrophysiology. 2015; 38 (8): 989-996. doi: 10.1111/pace.12660.

8. Koźluk, E., Zyśko, D., Piątkowska, A., Kiliszek, M., Lodziński, P., Matkowska, S., et al. Effectiveness comparison of various atrial fibrillation ablation methods in patients with common venous trunk. Advances in clinical and experimental medicine: official organ Wroclaw Medical University. 2018.

9. Shi, Y., Mi, S., Shi, Y., Wang, H., Li, J., Yu, B., et al. Evaluation of pulmonary vein anatomy using 256-slice computed tomography. Turkish journal of medical sciences. 2017; 47 (5): 1526-1534. doi: 10.2174/157340309787048121.

10. Odozynski, G., Forno, A. R. J. D., Lewandowski, A., Nascimento, H. G., \& d'Avila A. Paroxysmal Atrial Fibrillation Catheter Ablation Outcome Depends on Pulmonary Veins Anatomy. Arquivos brasileiros de cardiologia, (AHEAD). 2018. doi: 10.5935/abc. 20180069 .

11. Haissaguerre, M., Jaïs, P., Shah, D. C., Takahashi, A., Hocini, M., Quiniou, G., et al. Spontaneous initiation of atrial fibrillation by ectopic beats originating in the pulmonary veins. New England Journal of Medicine. 1998; 339 (10): 659-666. doi: 10.1056/NEJM199809033391003.

12. Steklov V. I., Morozov D. A., Emel'yanenko M. V., Kuz'menkov D. V. Psevdorotacionnaya atriografiya. Russian electronic journal of radiology 2015; 5 (1): 16-18. (In Russian)

13. Kashtanova N.Y., Gruzdev I.S., Kondrat'ev E.V., Artyukhina 


\section{RUSSIAN ELECTRONIC JOURNAL OF RADIOLOGY}

E.A., Yashkov M.V., Revishvili A.S. Cardiac multispiral computed tomography: optimization of examination's protocol for noninvasive cardaic mapping before catheter ablation of atrial fibrrillation. Medical Visualization. 2018; (3): 33-48. (In Russian) https://doi.org/10.24835/1607-0763-2018-3-33-48

14. Kiuchi, K., Yoshida, A., Takei, A., Fukuzawa, K., Itoh, M., Imamura, K., et al. Topographic variability of the left atrium and pulmonary veins assessed by $3 D-C T$ predicts the recurrence of atrial fibrillation after catheter ablation. Journal of arrhythmia. 2015; 31 (5): 286-292. doi: 10.1016/j.joa.2015.03.006.

15. Jongbloed, M. R., Dirksen, M. S., Bax, J. J., Boersma, E., Geleijns, K., Lamb, H. J., et al. Atrial fibrillation: multi-detector row CT of pulmonary vein anatomy prior to radiofrequency catheter ablation-initial experience. Radiology. 2005; 234 (3): 702 709. doi: 10.1148/radiol.2343031047.

16. Kanaji, Y., Miyazaki, S., Iwasawa, J., Ichihara, N., Takagi, T., Kuroi, A., et al. Pre-procedural evaluation of the left atrial anatomy in patients referred for catheter ablation of atrial fibrillation. Journal of cardiology. 2016; 67 (1): 115-121. doi: 10.1016/j.jjcc.2015.02.016.

17. Thorning, C., Hamady, M., Liaw, J. V. P., Juli, C., Lim, P. B., Dhawan, $R$., et al. CT evaluation of pulmonary venous anatomy variation in patients undergoing catheter ablation for atrial fibrillation. Clinical imaging. 2011; 35 (1): 1-9. doi: 10.1371/journal.pcbi.1006166.

18. Chen, J., Yang, Z. G., Xu, H. Y., Shi, K., Long, Q. H., \& Guo, $Y$. K. Assessments of pulmonary vein and left atrial anatomical variants in atrial fibrillation patients for catheter ablation with cardiac CT. European radiology. 2017; 27 (2): 660-670. doi: 10.1007/s00330-016-4411-6.

19. Klimek-Piotrowska, W., Hołda, M. K., Piatek, K., Koziej, M.,
\& Hołda, J. Normal distal pulmonary vein anatomy. 2016; 4: 1579. doi: 10.7717/peerj. 1579.

20. Bittner, A., Mönnig, G., Vagt, A. J., Zellerhoff, S., Wasmer, $K$., Köbe, J., et al. Pulmonary vein variants predispose to atrial fibrillation: a case-control study using multislice contrastenhanced computed tomography. Europace. 2011; 13 (10): 1394-1400. doi: 10.1093/europace/eur145.

21. Kato, R., Lickfett, L., Meininger, G., Dickfeld, T., Wu, R., Juang, G., et al. Pulmonary vein anatomy in patients undergoing catheter ablation of atrial fibrillation: lessons learned by use of magnetic resonance imaging. Circulation. 2003; 107 (15): 2004 2010. doi: 10.1161/01.CIR.0000061951.81767.4E.

22. Marom, E. M., Herndon, J. E., Kim, Y. H., \& McAdams, H. P. Variations in pulmonary venous drainage to the left atrium: implications for radiofrequency ablation. Radiology. 2004; 230 (3): 824-829. doi: 10.1148/radiol.2303030315.

23. Defaye, P., Kane, A., \& Jacon, P. An unusual connection of the right and left inferior pulmonary veins in the left atrium via a common ostium. Heart. 2010; 96 (23): 1951. doi: 10.1136/ha.2010.002899.

24. McLellan, A. J., Ling, L. H., Ruggiero, D., Wong, M. C., Walters, T. E., Nisbet, A., et al. Pulmonary vein isolation: the impact of pulmonary venous anatomy on long-term outcome of catheter ablation for paroxysmal atrial fibrillation. Heart Rhythm. 2014; 11 (4): 549-556. doi: 10.1016/j.hrthm.2013.12.025.

25. Wei, H. Q., Guo, X. G., Zhou, G. B., Sun, Q., Yang, J. D., Xie, H. Y., et al. Procedural findings and clinical outcome of second-generation cryoballoon ablation in patients with variant pulmonary vein anatomy. Journal of cardiovascular electrophysiology. 2018. 